\title{
Effect of exchange-correlation potential on the plasmon dispersions in a doped symmetrical double quantum well
}

\author{
E. Şenadım Tüzemen ${ }^{1}$, A. Turkoglu ${ }^{2}$, Y. Ergun ${ }^{*}, 3$, I. Sokmen ${ }^{4}$, and B. Tanatar ${ }^{5}$ \\ ${ }^{1}$ Cukurova University, Fac Scie \& Letters, Department of Physics, Adana 01330, Turkey \\ ${ }^{2}$ Cumhuriyet University, Department of Physics, 58140 Sivas, Turkey \\ ${ }^{3}$ Anadolu University, Department of Physics, 26470 Eskisehir, Turkey \\ ${ }^{4}$ Dokuzeylul University, Department of Physics, Tnaztepe-Izmir, Turkey \\ ${ }^{5}$ Department of Physics, Bilkent University, 06800 Bilkent, Ankara, Turkey
}

Received 22 May 2006, revised 14 August 2006, accepted 17 August 2006 Published online 27 September 2006

PACS 71.15.Ap, 71.15.Mb, 71.45.Gm, 71.55.-i, 73.21.Fg

We have calculated the plasmon dispersion relations in a doped double quantum well with and without exchange-correlation potential added to the effective potential of the system. The calculations were done for high and low doping densities by solving the Schrödinger and Poisson equations self-consistently. Our numerical results show that the exchange-correlation potential is quite important at high doping densities of donor impurities for plasmon dispersions at large wave vectors. On the other hand, the ratio of subband populations $n_{i}$ to donor density $N_{\mathrm{D}}$ is more affected at low densities.

(c) 2007 WILEY-VCH Verlag GmbH \& Co. KGaA, Weinheim

\section{Introduction}

Double quantum well systems offer a useful model to understand tunneling-time of carriers through the barriers, and electron-electron interaction induced carrier relaxation of adjacent atoms in solids and nonideal gases which are known to be important processes in field-effect transistors (FET) [1], quantum well cascade lasers (QCL), and quantum well infrared photodetectors (QWIP). Intersubband electronelectron interaction in symmetric and asymmetric double quantum well structures is theoretically studied by Marcos et al. $[2,3]$ using GW approximation with inelastic scattering rate in a two-subband model. In QCL and QWIP, while the intersubband inelastic relaxation turns out to be the primary rate-limiting scattering process, in the planar hot electron transistors with related two dimensional (2D) high speed devices, intra-subband relaxation due to electron-electron interaction is pointed out to be very important.

In this work, we calculate the plasmon dispersion relations for the in-phase and out-of-phase modes in doped symmetrical double quantum wells comparing the effect of exchange-correlation potential. Our motivation comes from a number of recent studies addressing the plasmon dispersions in double quantum well systems [4-7] and given the wide device applications [8-10] as mentioned above we would like to assess the importance of exchange-correlation effects in these structures. The composition of the system is designed from GaAs-GaAlAs structures. Barriers surrounding the wells were doped to investigate effect of the exchange-correlation potential.

\footnotetext{
${ }^{*}$ Corresponding author: e-mail: yergun@anadolu.edu.tr, Phone: +90 2223350580
} 
In the next section we briefly describe the theoretical method with which we calculate the plasmon dispersions. Sample parameters of the system are also given in the same section. Our numerical results are presented and a discussion is followed in Section 3.

\section{Theory}

Quantum wells and barriers used in this work are chosen to be GaAs and $\mathrm{Ga}_{0.67} \mathrm{Al}_{0.33} \mathrm{As}$, respectively. Two different doping (donor) densities were used to compare the effect of exchange-correlation potential on the plasmon dispersion relations. A small region (spacer) between wells and doped barriers, at the barrier side, is not doped to prevent positive (donor) and negative (electron) charge interaction. Onedimensional Schrödinger equation for carrier localization/growth direction is

$$
\left[-\frac{\hbar^{2}}{2 m^{*}} \frac{\partial^{2}}{\partial z^{2}}+V_{\text {eff }}(z)\right] \phi_{n}=E_{n} \phi_{n}
$$

and the effective potential is

$$
V_{\mathrm{eff}}=V_{\mathrm{conf}}(z)+V_{\mathrm{H}}(z)+V_{\mathrm{xc}}(z),
$$

where $V_{\text {conf }}$ is the confining potential, $V_{\mathrm{H}}$ and $V_{\mathrm{xc}}$ are the Hartree and exchange-correlation potentials, respectively, and $\phi_{n}$ are the subband wavefunctions. The Hartree and exchange-correlation potentials are obtained from the charge distribution $n(z)$, such that

$$
V_{\mathrm{H}}(z)=-\frac{4 \pi e^{2}}{\varepsilon}\left[n(z)-N_{D}(z)\right] .
$$

For the exchange-correlation potential we use the local-density approximation introduced by Hedin and Lundqvist [11]

$$
V_{\mathrm{xc}} \approx \mu_{\mathrm{xc}}=\varepsilon_{\mathrm{xc}}+n \frac{\mathrm{d} \varepsilon_{\mathrm{xc}}}{\mathrm{d} n}
$$

where $\mu_{\mathrm{xc}}$ is the exchange-correlation part of the chemical potential and $\varepsilon_{\mathrm{xc}}$ is the exchange-correlation energy of a uniform electron gas. Hedin and Lundqvist [11] write $\mu_{\mathrm{xc}}$ in terms of the exchange part $\mu_{\mathrm{x}}$ and a correlation enhancement factor $\beta$, so that $\mu_{\mathrm{xc}}(n)=\beta(n) \mu_{x}(n)$, where $\mu_{\mathrm{x}}=(3 n)^{1 / 2} / 2 \pi e^{2} / 4 \pi \varepsilon_{0}$ and $\beta=1+B x \ln (1+1 / x)$. Here, $x=(4 \pi n / 3)^{-1 / 3} / A a_{\mathrm{B}}^{* \mathrm{xc}}$ and $A=21$ and $B=0.7734$ are numerical constants chosen to reproduce the correlation energy of the uniform electron gas. Combining the above we obtain

$$
V_{\mathrm{xc}}=-0.985 \frac{e^{2}}{4 \pi \varepsilon_{0}} n^{1 / 3}(z)\left\{1+\frac{0.0334}{a_{\mathrm{B}}^{*} n^{1 / 3}(z)} \ln \left[1+18.376 a_{\mathrm{B}}^{*} n^{1 / 3}(z)\right]\right\},
$$

in which $a_{\mathrm{B}}^{*}$ is the effective Bohr radius for electron effective mass $m^{*}$ and background dielectric constant $\varepsilon_{0}$. The exchange-correlation potential is that given by a perturbative calculation which has been parameterized as an analytic expression in the local-density approximation [11]. Although there are a number of more recent exchange-correlation potentials being used in the Ref. [12], we found it sufficient to use the above form at the densities considered in this work.

To calculate the plasmon dispersions, we need to solve for the poles of the polarization function (in matrix form)

$$
\Pi(q, \omega)=\left[1-\left(V^{\mathrm{H}}(q)-V^{\mathrm{xc}}(q)\right) \Pi^{0}(q, \omega)\right]^{-1} \Pi^{0}(q, \omega),
$$


where $V^{\mathrm{H}}(q)$ and $V^{\mathrm{xc}}(q)$ are the static ( $q$-dependent) Fourier transforms of the bare Coulomb interaction and the vertex function, respectively given as $[13,14]$

$$
V_{i j k l}^{\mathrm{H}}(q)=\frac{2 \pi e^{2}}{q \varepsilon} \int \mathrm{d} z \mathrm{~d} z^{\prime} \phi_{i}(z) \phi_{j}(z) \mathrm{e}^{-q\left|z-z^{\prime}\right|} \phi_{k}\left(z^{\prime}\right) \phi_{l}\left(z^{\prime}\right),
$$

and

$$
V_{i j k l}^{\mathrm{xc}}=-\int \mathrm{d} z \mathrm{~d} z^{\prime} \phi_{i}(z) \phi_{j}(z) \frac{\partial V_{\mathrm{xc}}(z)}{\partial n} \phi_{k}\left(z^{\prime}\right) \phi_{l}\left(z^{\prime}\right) .
$$

The above matrix elements are calculated using the self-consistently determined subband wave functions $\phi_{i}(z)$. Finally, $\Pi^{0}(q, \omega)$ in Eq. (6) is the two-dimensional noninteracting polarizability. We follow the analyses of previous works $[3,5-7]$ to calculate the plasmon dispersions by solving for the zeros of the dielectric matrix $\varepsilon(q, \omega)$, with and without the exchange-correlation effects by including and omitting $V_{\mathrm{xc}}$, respectively. Our numerical results are presented in the next section.

\section{Numerical results and discussion}

We consider two coupled symmetric quantum wells of width $L=100 \AA$ and the barrier width is $L_{\mathrm{b}}=25 \AA$. Doping spacers are taken to be $10 \AA$ to prevent ionized donor-confined electron interaction. Fermi level is taken as $70 \mathrm{meV}$ below the conduction band of barriers surrounded by the wells while the conduction band is flat. This value of Fermi level coincides with the binding energy of the donor atoms inside the wells [6]. The system is investigated under two different doping densities to obtain the subband wavefunctions from which the plasmon dispersion (the localized donor charges are assumed to be smeared out uniformly in the plane) are calculated. We perform calculations with and without the exchange-correlation potential to assess its influence.

Conduction band profile of the quantum-well structure was obtained for different both doping concentrations solving the Poisson and Schrödinger equations self-consistently developed by the present authors [15]. Calculated band profile of the structure and their localized wavefunctions are shown in Fig. 1(a)(b) for doping density $N_{\mathrm{D}}=5 \times 10^{18} \mathrm{~cm}^{-3}$. While Fig. 1(a) does not include the effect of exchange-

$\mathrm{eV}$

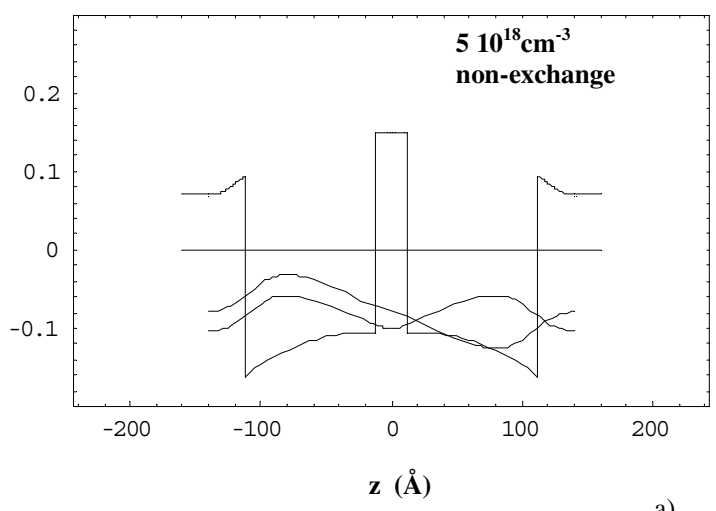

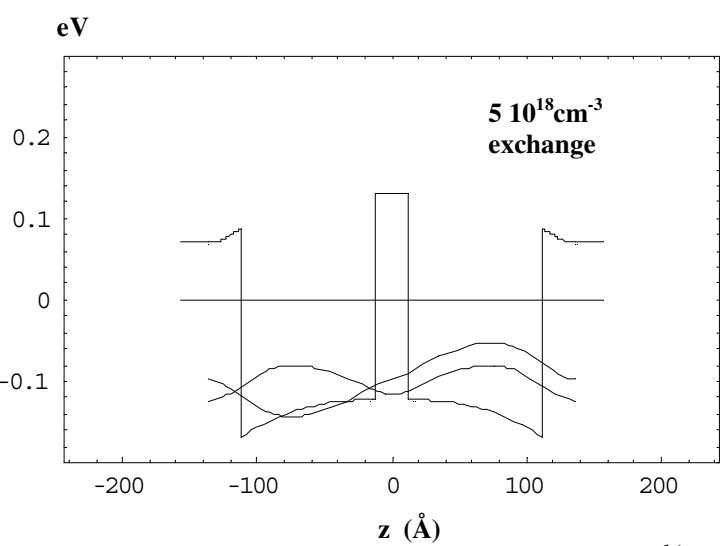

b)

Fig. 1 Effective potential, Fermi energy (horizontal straight line), and subband profile of GaAs-AlGaAs double quantum well system for $N_{\mathrm{D}}=5 \times 10^{18} \mathrm{~cm}^{-3}$ : (a) exchange-correlation effects are included in the self-consistent calculations, (b) no exchange-correlation effects. 

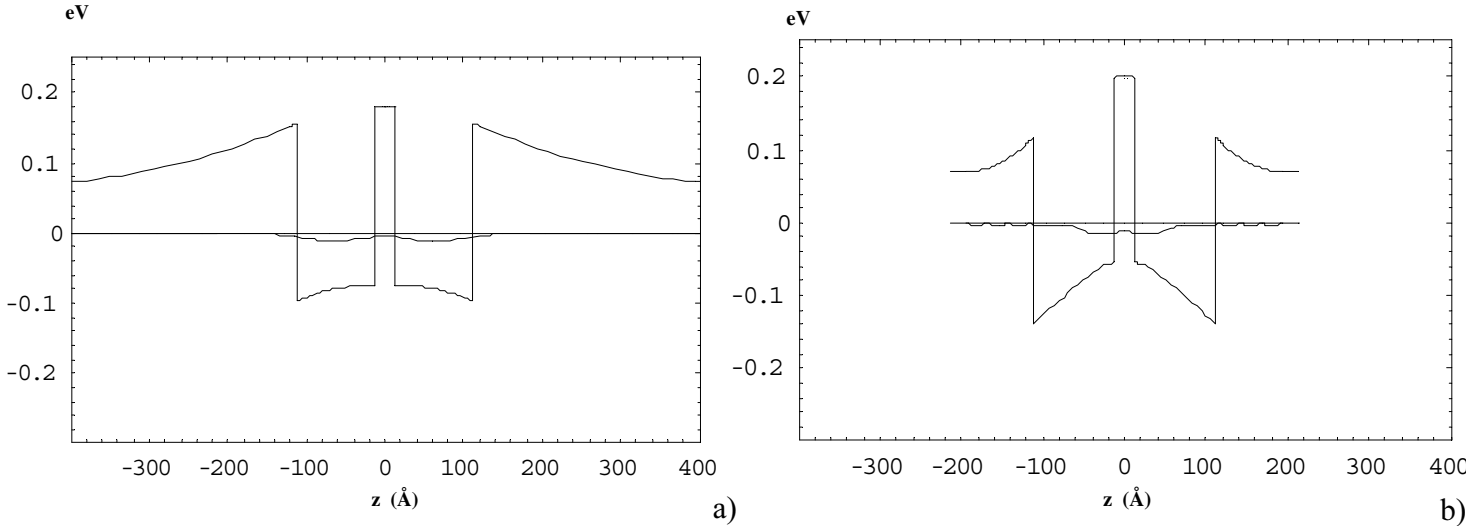

Fig. 2 a) Potential profile of DQW and exchange-correlation potential for the doping density of donor atoms is $10^{17} \mathrm{~cm}^{-3}$. Exchange-correlation potential is seen as doublet pockets in figure. (b) Potential profile of DQW and exchange-correlation potential for the doping density of donor atoms is $10^{18} \mathrm{~cm}^{-3}$. Exchange-correlation potential is seen as doublet pockets in the figure but centered at the origin of the coordinate axes.

correlation potential, Fig. 1(b) presents the effect of exchange-correlation potential at the same doping density. Figure 2(a) shows the potential profile of double quantum wells with the exchange correlation potential obtained by the Eq. (5) for the $10^{17} \mathrm{~cm}^{-3}$ doping density of donor atoms while Fig. 2(b) for $10^{18} \mathrm{~cm}^{-3}$. Confinement of the carriers in the wells increase when doping density increases but the exchange-correlation potential tends preventing of this effect by degreasing the slope of the triangular wells seen in Fig. 2(b). On the other hand, because the localization energy goes away from the Fermi energy level when doping density increases (solid line drawn at energy zero in figures) and also two dimensional carrier density depends on the $\left(\varepsilon-\varepsilon_{\mathrm{F}}\right)$, centered exchange-correlation potential increases the tunneling probability of carriers and thus increases two dimensional carrier density by going away below from the Fermi level. As a result of the explanations given above, exchange-correlation potential valleys shift to $z=0$ region at the both sides.

Similarly, Fig. 3(a)-(b) displays the conduction band profiles of our structure with and without the exchange-correlation potential included in the calculations at the lower doping density of $N_{\mathrm{D}}=5 \times 10^{17} \mathrm{~cm}^{-3}$.
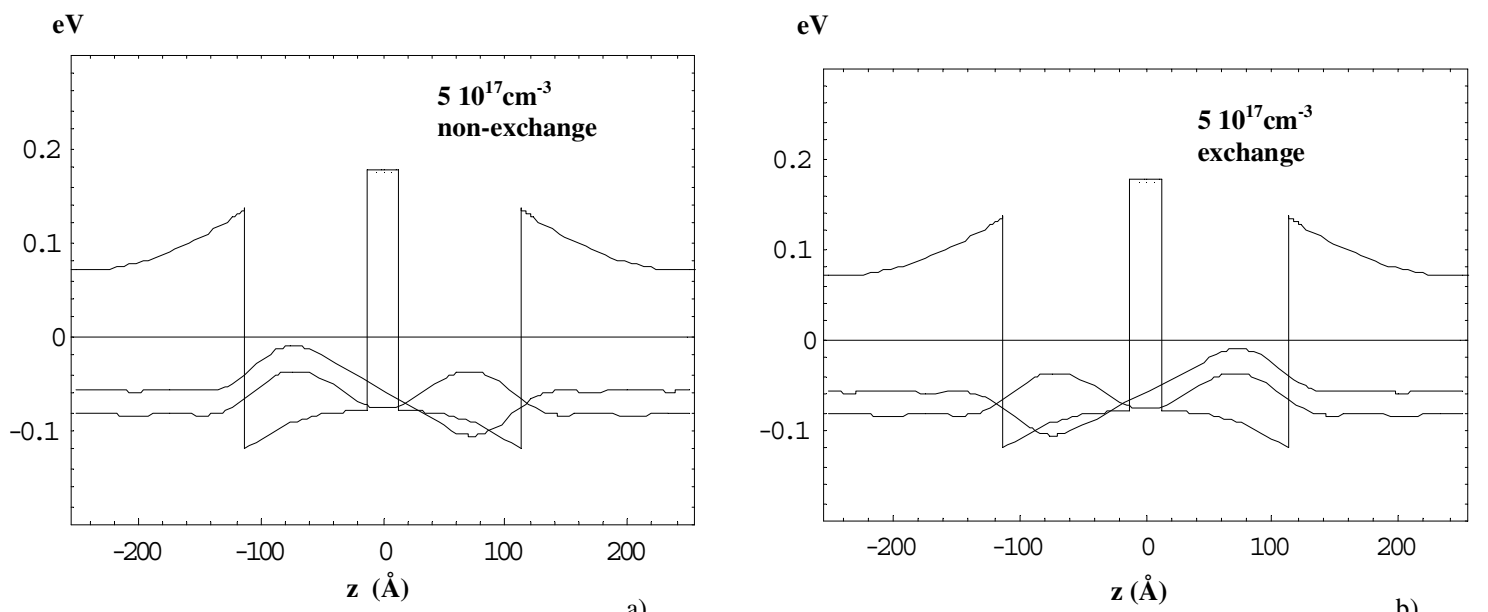

Fig. 3 Subband profile of GaAs-GaAlAs double quantum well system for $N_{\mathrm{D}}=5 \times 10^{17} \mathrm{~cm}^{-3}$ : (a) exchangecorrelation effects are included in the self-consistent calculations, (b) no exchange-correlation effects. 


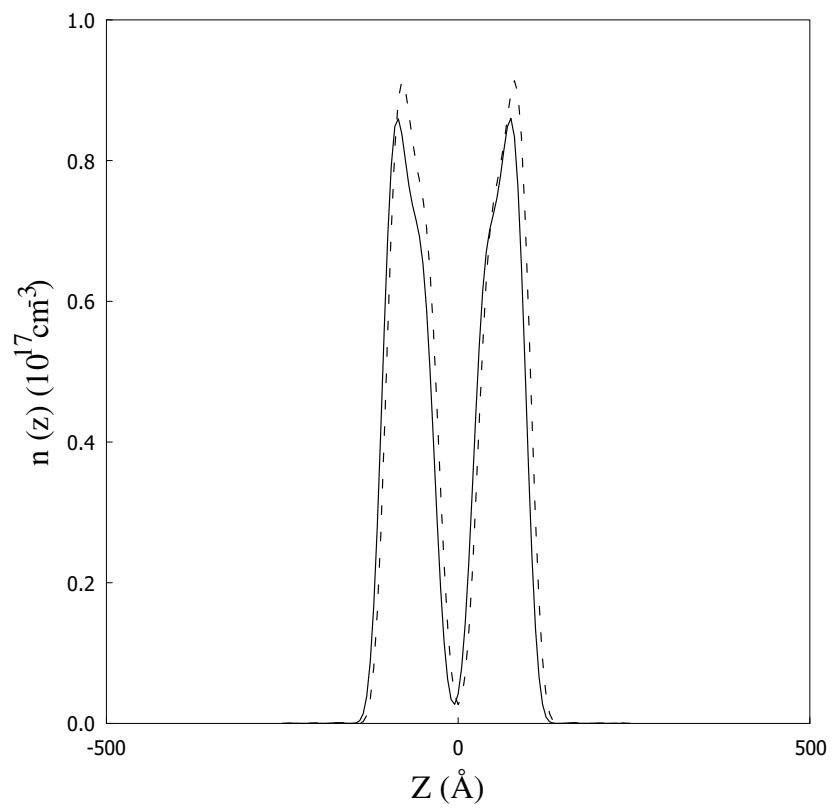

Fig. 4 Electron density profiles with (dashed line) and without (solid line) exchange-correlation effects for $N_{\mathrm{D}}=5 \times 10^{17} \mathrm{~cm}^{-3}$.

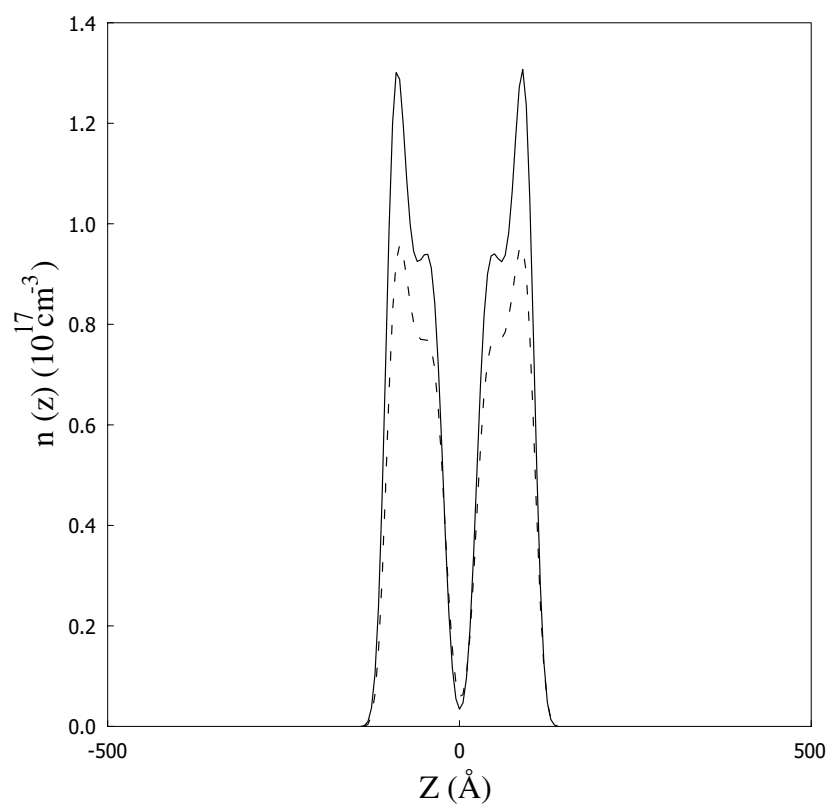

Fig. 5 Electron density profiles with (dashed line) and without (solid line) exchange-correlation effects for $N_{\mathrm{D}}=5 \times 10^{18} \mathrm{~cm}^{-3}$. 


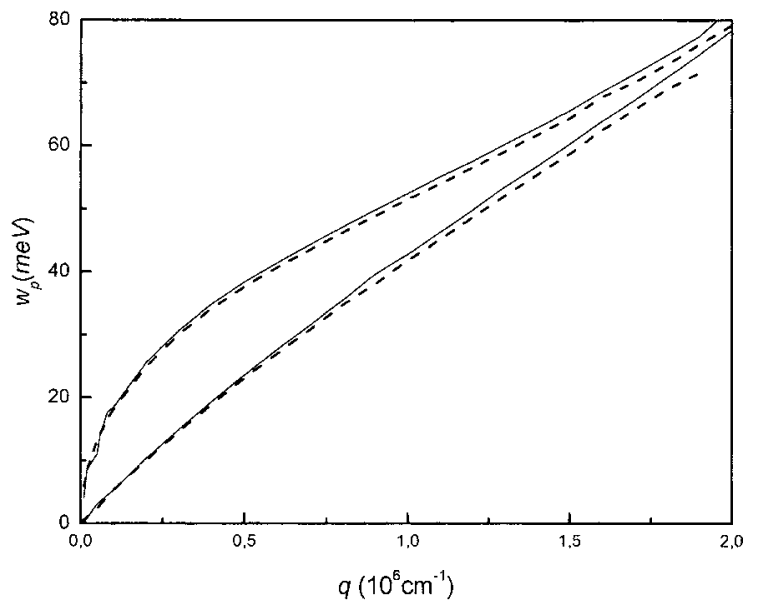

Fig. 6 Plasmon dispersions with (solid line) and without (dashed line) exchange-correlation effects for $N_{\mathrm{D}}=5 \times 10^{17} \mathrm{~cm}^{-3}$.

Total carrier distribution in the symmetrical double quantum well is shown in Fig. 4(a)-(b) and Fig. 5(a)-(b) for these different doping densities. At high donor concentrations, an increasing charge density in the confining potential leads to more band bending and gives rise to the formation of a deeper quantum well (see Figs. 1-3 and Ref. [16] for detailed information on symmetric and doped double quantum well systems).

We present our results for the plasmon dispersions in the examined double quantum well structures in Figs. 6 and 7. We observe the usual "optical" branch corresponding to the in-phase oscillations of the electrons (upper curves) and "acoustic" branch corresponding to the out-of-phase oscillations (lower curves). Because of the finite barrier height and thickness, the acoustic branch actually has a gap at long wavelength $(q \rightarrow 0)$ which is hardly noticeable at the scale we present them. With decreasing barrier height and thickness the tunneling effects become more appreciable and the plasmon gap grows to become more significant. These results are consistent with the long wavelength behavior of plasmons in double quantum well systems [4-7]. As we are interested in the effects of exchange-correlation potential on the plasmon dispersions we concentrate on the large wave vector region. Figures 6 and 7 show plasmon dispersion relation for low and high doping densities of donor atoms. In these Figs. calculations with (solid lines) and without (dashed lines) the exchange-correlation (xc) potential are presented. As depicted in Figs. 5 and 6, xc effects are important for plasmon dispersion at higher doping densities. On the other hand, relative change in the subband population depending on the xc potential shows an opposite behavior. Since the electron density in the $i$-th subband $n_{i}=\left(m^{*} / \pi \hbar^{2}\right)\left(E_{\mathrm{F}}-E_{i}\right)$ depends on the

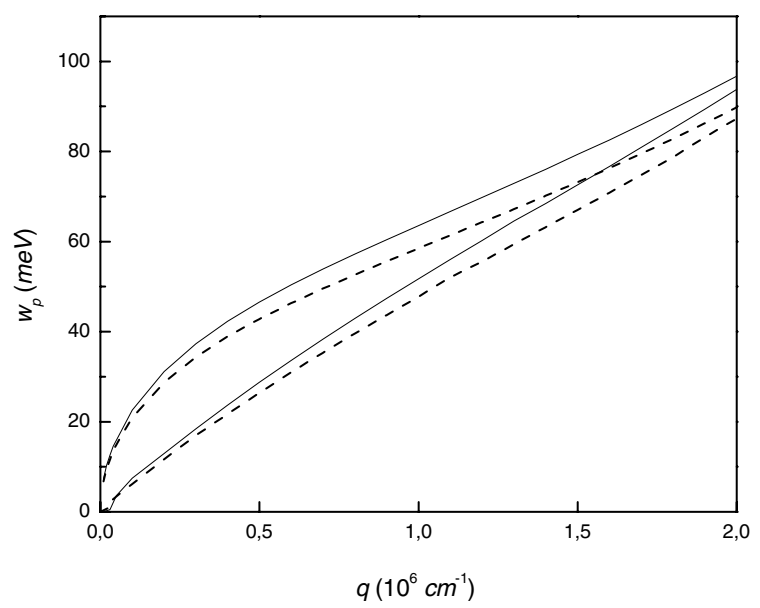

Fig. 7 Plasmon dispersions with (solid line) and without (dashed line) exchange-correlation effects for $N_{\mathrm{D}}=5 \times 10^{18} \mathrm{~cm}^{-3}$. 
Fermi energy or equivalently the Fermi wave vector, to quantify this effect we have looked at the Fermi vectors for the two doping densities under investigation. For the first or second subband, the difference between the Fermi wave vectors with and without the xc potential is $k_{\mathrm{F}}(\mathrm{xc})-k_{\mathrm{F}}(\mathrm{nxc})=0.06 \times 10^{6} \mathrm{~cm}^{-1}$ for the doping density $5 \times 10^{17} \mathrm{~cm}^{-3}$. The same quantity yields about $0.26 \times 10^{6} \mathrm{~cm}^{-1}$ for the doping density $5 \times 10^{18} \mathrm{~cm}^{-3}$.

\section{Conclusions}

We have performed calculations to discern the effect of exchange-correlation potential on the plasmon dispersions of doped double quantum well systems and found that it is more significant in higher doping concentrations. This should be useful in device applications where plasmon effects are important for especially double quantum well field effect transistors at high density doping limits.

Acknowledgments The authors would like to thank The State Planning Organization of Turkey (DPT) (Project no: DPT-K. 120220.2005) and Anadolu University for funding of this project. B.T. gratefully acknowledges the support of TUBITAK and TUBA.

\section{References}

[1] X. G. Peralta, S. J. Allen, M. C. Wanke, N. B. Harff, J. A. Simmons, M. P. Lilly, J. L. Reno, P. J. Burke, and J. P. Eisentein, Appl. Phys. Lett. 81, 1627 (2002).

[2] M. R. S. Tavares and G. Q. Hai, Physica E 13, 794 (2002).

[3] M. R. S. Tavares, G. Q. Hai, and S. Das Sarma, Phys. Rev. B 64, 045325 (2001).

[4] S. Das Sarma and E. H. Hwang, Phys. Rev. Lett. 81, 4216 (1998); Phys. Rev. B 63, 233201 (2001).

[5] G. Gumbs and G. R. Aizin, Phys. Rev. B 51, 7074 (1995).

[6] X.-H. Liu, X.-H. Wang, and B. Y. Gu, Phys. Rev. B 64, 195322 (2001).

[7] M.-T. Bootsmann, C.-M. Hu, Ch. Heyn, D. Heitmann, and C. Schüller, Phys. Rev. B 67, 121309 (2003). S. Holland, C.-M. Hu, Ch. Heyn, and D. Heitmann, Phys. Rev. B 66, 073305 (2002).

[8] M. Lee, M. C. Wanke, and J. L. Reno, Appl. Phys. Lett. 86, 033501 (2005).

[9] V. V. Popov, T. V. Teperik, Y. N. Zayko, S. T. Alten, and N. J. M. Horing, Solid State Electron. 49, 1049 (2005).

[10] S. M. Maung and S. Katayama, J. Phys. Soc. Jpn. 73, 2562 (2004).

[11] L. Hedin and B. I. Lundqvist, J. Phys. C 4, 2064 (1971).

[12] Q. Zhao, R. C. Morrison, and R. G. Parr, Phys. Rev. A 50, 2138 (1994).

[13] R. J. Radtke, P. I. Tamborenea, and S. Das Sarma, Phys. Rev. B 54, 13832 (1996).

[14] B. Y. K. Hu and S. Das Sarma, Phys. Rev. B 48, 5469 (1993).

[15] E. Ozturk, Y. Ergun, H. Sari, and I. Sokmen, Appl. Phys. A 73, 749 (2001).

[16] F. Ungan, E. Ozturk, Y. Ergun, and I. Sokmen, Eur. Phys. J. Appl. Phys. 29, 27 (2005). 